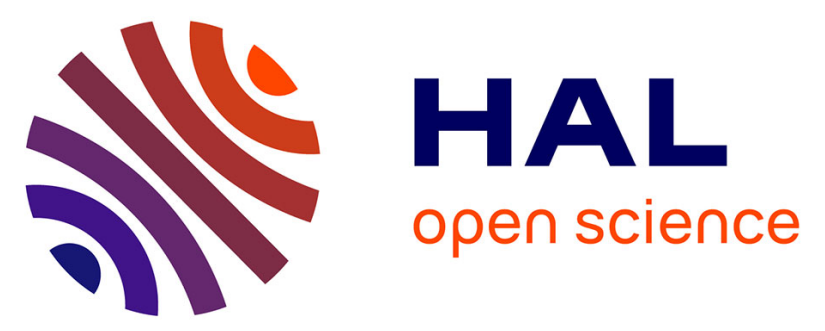

\title{
From core-shell BaTiO3@MgO to nanostructured low dielectric loss ceramics by spark plasma sintering
}

Romain Berthelot, Brice Basly, Sonia Buffière, Jérôme Majimel, Geoffroy

Chevallier, Alicia Weibel, Amélie Veillère, Laetitia Etienne, U-Chan Chung, Graziella Goglio, et al.

\section{To cite this version:}

Romain Berthelot, Brice Basly, Sonia Buffière, Jérôme Majimel, Geoffroy Chevallier, et al.. From core-shell BaTiO3@MgO to nanostructured low dielectric loss ceramics by spark plasma sintering. Journal of Materials Chemistry C, 2014, 2 (4), pp.683-690. 10.1039/c3tc31990c . hal-01130844

\section{HAL Id: hal-01130844 \\ https://hal.science/hal-01130844}

Submitted on 12 Mar 2015

HAL is a multi-disciplinary open access archive for the deposit and dissemination of scientific research documents, whether they are published or not. The documents may come from teaching and research institutions in France or abroad, or from public or private research centers.
L'archive ouverte pluridisciplinaire HAL, est destinée au dépôt et à la diffusion de documents scientifiques de niveau recherche, publiés ou non, émanant des établissements d'enseignement et de recherche français ou étrangers, des laboratoires publics ou privés. 


\section{OATAO}

\section{Open Archive TOULOUSE Archive Ouverte (OATAO)}

OATAO is an open access repository that collects the work of Toulouse researchers and makes it freely available over the web where possible.

This is an author-deposited version published in : http://oatao.univ-toulouse.fr/ Eprints ID : 13631

To link to this article : DOI:10.1039/c3tc31990c

URL : http://dx.doi.org/10.1039/c3tc31990c

\section{To cite this version :}

Berthelot, Romain and Basly, Brice and Buffière, Sonia and Majimel, Jérôme and Chevallier, Geoffroy and Weibel, Alicia and Veillère, Amélie and Etienne, Laetitia and Chung, U-Chan and Goglio, Graziella and Maglione, Mario and Estournès, Claude and Mornet, Stéphane and Elissalde, Catherine From core-shell BaTiO3@MgO to nanostructured low dielectric loss ceramics by spark plasma sintering. (2014) Journal of Materials Chemistry. C, vol. 2 ( $\mathrm{n}^{\circ}$ 4). pp. 683-690. ISSN 2050-7526

Any correspondance concerning this service should be sent to the repository administrator: staff-oatao@ listes-diff.inp-toulouse.fr 


\title{
From core-shell $\mathrm{BaTiO}_{3} \mathrm{aMgO}$ to nanostructured low dielectric loss ceramics by spark plasma sintering $\dagger$
}

\author{
Romain Berthelot, ${ }^{a b}$ Brice Basly, ${ }^{a b}$ Sonia Buffière, ${ }^{a b}$ Jérôme Majimel, ${ }^{a b}$ \\ Geoffroy Chevallier, ${ }^{c d}$ Alicia Weibel, ${ }^{c d}$ Amélie Veillère, ${ }^{a b}$ Laëtitia Etienne, $^{\text {ab }}$ \\ U-Chan Chung, ${ }^{\text {ab }}$ Graziella Goglio, ${ }^{\text {ab }}$ Mario Maglione, ${ }^{\text {ab }}$ Claude Estournès, ${ }^{\text {cd }}$ \\ Stéphane Mornet ${ }^{\star a b}$ and Cathy Elissalde ${ }^{\star a b}$
}

\begin{abstract}
We report a quite general way to design materials with tailored properties by combining thermolysis and fast sintering approaches. Submicrometric-sized $\mathrm{BaTiO}_{3}$ particles have been directly coated in a continuous nanocrystalline $\mathrm{MgO}$ shell through a thermal decomposition process. The electron microscopy study has evidenced a shell composed of randomly oriented $\mathrm{MgO}$ nanocrystallites. The final nanostructured composite, made of sub-micrometric $\mathrm{MgO}$ and $\mathrm{BaTiO}_{3}$ grains uniformly distributed, is obtained in situ during the SPS process. Such a rearrangement can be explained by the initial core-shell architecture, the weak cohesion of the $\mathrm{MgO}$ nanocrystallites and their soft plastic behavior under SPS conditions. The composite effect leads to significant modifications in both the dielectric properties and Curie-Weiss parameters compared to uncoated $\mathrm{BaTiO}_{3}$, especially a decrease and thermal stabilization of both the permittivity and the dielectric losses. We ascribe such changes to the stress generated during SPS through the extended interfaces between the two components
\end{abstract}

\section{Introduction}

The search for functional materials in numerous fields of applications has led to the development of composite materials combining the nominal behaviour of each constituent to give the finally obtained tailored properties. With the positive input of surface and colloidal sciences, the composite architecture can be now controlled down to the nanoscale. The coating of (nano)particles to form core-shell morphologies has been extensively used to combine singular physical properties of each component and led to significant advances in functional

${ }^{a}$ Univ. Bordeaux, ICMCB, UPR 9048, F-33600 Pessac, France. E-mail: mornet@ icmcb-bordeaux.cnrs.fr; elissald@icmcb-bordeaux.cnrs.fr; Fax: +33 540002761; Tel: +33540006335; +33540002696

${ }^{b} I C M C B-C N R S$, UPR 9048, 87 avenue du Dr Albert Schweitzer, F-33600 Pessac, France 'Université de Toulouse, UPS, INP, Institut Carnot Cirimat, 118 route de Narbonne, F-31062 Toulouse cedex 9, France

${ }^{d}$ CNRS, Institut Carnot Cirimat, F-31062 Toulouse, France

$\dagger$ Electronic supplementary information (ESI) available: Bright-field TEM micrographs of $\mathrm{BaTiO}_{3}-\mathrm{MgO}$ particles obtained after the washing sequence with cyclohexane only (Fig. S1). In situ dilatometry curves obtained during the SPS densification of the composite and pure BT ceramics (Fig. S2). An enlarged view of XRD powder patterns of the raw BT, sintered BT, as-prepared BT@MgO, and $\mathrm{BT} @ \mathrm{MgO}$ after the heat treatment for $1 \mathrm{~h}$ at $600{ }^{\circ} \mathrm{C}$, and the sintered composite ceramic (Fig. S3). Temperature evolution of the inverse of permittivity $\left(1 / \varepsilon^{\prime}\right)$ measured for the composite and BT SPS ceramics in addition to the corresponding Curie-Weiss fitting in the paraelectric domain (Fig. S4). See DOI: $10.1039 / \mathrm{c} 3 \mathrm{tc} 31990 \mathrm{c}$ materials such as photonic crystals, biomedical magnetic or drug delivery systems. ${ }^{1-3}$

However, the coating of grains by an oxide shell is emerging in the field of ferroelectric materials. ${ }^{4-7}$ Driven by the need of functional materials for advanced electronic systems, such as multilayer ceramic capacitors and tunable devices, composite materials combining a ferroelectric oxide (mainly $\mathrm{BaTiO}_{3}$ (BT) or $\mathrm{Ba}_{1-x} \mathrm{Sr}_{x} \mathrm{TiO}_{3}$ (BST) perovskites) with a non-ferroelectric one are the focus of research interest in order to obtain functional ceramics with tailored dielectric properties. As first examples, Buscaglia and coworkers directly grew $\mathrm{ABO}_{3}$ perovskites $\left(\mathrm{SrTiO}_{3}\right.$ and $\left.\mathrm{BaZrO}_{3}\right)$ or various binary oxides $\left(\mathrm{Y}_{2} \mathrm{O}_{3}, \mathrm{NiO}\right.$, etc. $)$ on nanosized BT particles through precipitation methods. ${ }^{\mathbf{4} \mathbf{6}}$ Silica coating has also been recently applied to ferroelectric particles in order to reduce the dielectric losses. Indeed a controlled growth of the silica shell on BT or BST nanoparticles has been already performed to obtain core-shell particles used as precursors of composite ceramics. ${ }^{5,8-10}$ However, the main drawback of silica is that the sintering temperature cannot be increased to improve densification because of the interphase formation occurring at the early stage of the sintering process. ${ }^{\mathbf{1 1}}$

An interesting alternative dielectric oxide is $\mathrm{MgO}$, thanks to its highly refractory properties allowing higher processing temperatures. Therefore, $\mathrm{MgO}$ has been already combined with ferroelectric materials to obtain composite materials with tailored properties. ${ }^{12}$ For example multilayers as well as $3 \mathrm{D}$ dense ceramics made of BST ferroelectric and $\mathrm{MgO}$ dielectric 
oxides with fully controlled interfaces exhibit promising tailored dielectric properties, especially with very low dielectric losses. ${ }^{13-15}$ At the nanoscale, the search for coating of $\mathrm{MgO}$ onto ferroelectric particles has received particular interest in the last decade, especially by the use of hydrothermal, sol-gel or precipitation routes. ${ }^{\mathbf{1 6 - 2 1}}$

In this work, a method based on the thermal decomposition of organometallic precursors is followed to prepare an efficient coating of BT ferroelectric particles by the crystalline MgO shell. The thermolysis technique is widely used for the synthesis of nanoparticles and core-shell nanoparticles with a wide range of chemical compositions, mainly metal oxides, chalcogenides or pure metals and alloys. ${ }^{3}$ The second motivation is the recent report of a high-quality $\mathrm{MgO}$ shell surrounding FePt nanoparticles. Indeed in their search for physical barriers preventing metallic particles from sintering under high temperature conditions, Kim et al. managed to coat ferromagnetic FePt particles by a robust $\mathrm{MgO}$ shell produced from the thermal decomposition of magnesium acetylacetonate $\left(\mathrm{Mg}(\mathrm{acac})_{2}\right){ }^{22,23}$ Until now and to the best of our knowledge, only metallic particles with a small mean diameter (less than $10 \mathrm{~nm}$ ) have been embedded by this method. In the present work, a complete shell of crystallized $\mathrm{MgO}$ nanoparticles is successfully obtained all around BT particles through this assembly process. The shell morphology has been deeply investigated by various high resolution microscopy techniques including chemical and structural probes, and an assembly mechanism is proposed.

In addition, among all the previous studies on BT@MgO or BST@MgO composite ceramics and with the notable exception of the work of Chen et al., ${ }^{19}$ only conventional sintering methods have been carried out to obtain the composite ceramic. However, in order to control the interfaces in the coreshell architecture, conventional sintering is now more and more frequently substituted by spark plasma sintering (SPS). Indeed such a densification method has been used to avoid grain growth and to limit the interdiffusion at the interface between the core and the shell in order to create locally graded structures, ${ }^{4}$ to control in situ solid state reaction in ferroelectric/ magnetic multifunctional composites, ${ }^{24}$ or to prevent interdiffusion in ferroelectric/dielectric ones. ${ }^{\mathbf{1 3 1 4}}$ Therefore by combining colloidal chemistry and fast sintering approaches, composite ceramics exhibiting tailored dielectric properties can be obtained.

In our study, the prepared $\mathrm{BaTiO}_{3} @ \mathrm{MgO}$ core-shell particles are used as precursors of composite ceramics densified by SPS. The weak cohesion of nanocrystalline MgO constituting the shell allows particle rearrangement during the sintering process, which leads in the final stage to a uniform distribution of $\mathrm{MgO}$ grains among the BT ones. The soft plastic behaviour of $\mathrm{MgO}$ under SPS conditions enables the enhancement of densification kinetics compared to uncoated BT and in addition the dielectric phase prevents ferroelectric grain growth. As a result of this nanostructure, both $\mathrm{BT}$ and $\mathrm{MgO}$ are uniformly and randomly distributed within the composite material. No interdiffusion is evidenced despite the high density of interfaces between the two components. The dielectric properties of the ceramics reveal a significant influence of the composite effect with especially very low and constant dielectric losses and modified Curie-Weiss parameters compared with the uncoated BT reference sintered under the same conditions.

\section{Experimental}

\subsection{Stabilization of BT particle dispersions in organic solvents}

BT nanoparticles with average diameter close to $300 \mathrm{~nm}$ were purchased from Sakai Chemical Industry Co. In order to stabilize dispersions of BT particles, a preliminary surface activation must be carried out. Particles were first dispersed by ultrasonic treatment in dilute $\mathrm{HNO}_{3}$ solution (Scharlau, reagent grade). After removal of the supernatant by centrifugation at $6000 \mathrm{~g}$ for 5 min and washing with deionized water, particles were dispersed in citric acid solution (Sigma Aldrich, reagent grade). They were again sonicated, centrifuged and washed under the same conditions. At the end of the washing step, the particles were dispersed in minimum volume of water and peptidized by addition of droplets of ammonia before dilution in ethanol. Then, ethanol was washed away from the BT particle dispersion by centrifugation ( $5 \mathrm{~min}$ at $6000 \mathrm{~g}$ ). BT particles were then dispersed in a volume of oleic acid solution in chloroform (typically $5 \mathrm{~mL}$, Sigma Aldrich, $\geq 99 \%$ containing $100 \mu \mathrm{L}$ of oleic acid for $1 \mathrm{~g}$ of starting BT powder).

\subsection{Coating of BT particles by MgO nanocrystallites}

For $1 \mathrm{~g}$ of BT particles with activated surface, $2.4 \mathrm{~g}$ of $\mathrm{Mg}(\mathrm{acac})_{2}$ (Strem Chemicals, 98\%) and $4.2 \mathrm{~g}$ of tetradecanediol (Sigma Aldrich, technical grade) were dissolved in a $100 \mathrm{~mL}$ roundbottom flask containing $60 \mathrm{~mL}$ of benzyl ether (Sigma Aldrich, $\geq 98 \%$ ) and $4 \mathrm{~mL}$ of oleic acid (Sigma Aldrich, $\geq 99 \%$ ) by heating at $80{ }^{\circ} \mathrm{C}$ for $30 \mathrm{~min}$ under magnetic stirring. The previously prepared BT-oleate nanoparticle dispersion in chloroform was then added into the flask, which was subsequently heated to $120{ }^{\circ} \mathrm{C}$ for at least $2.5 \mathrm{~h}$ under an open atmosphere, still under magnetic stirring, to fully evaporate the chloroform. The flask was then heated at $290{ }^{\circ} \mathrm{C}$ under refluxing conditions and under an Ar atmosphere. After 2.5 hours, the heating mantle was removed for slow cooling down to room temperature.

Finally, the nanoparticles were separated and purified using a mixture of cyclohexane and absolute ethanol (in $1: 7$ volume ratio, Sigma Aldrich, $99.7 \%$ and Scharlau, analytical grade, respectively) and low centrifugation sequences (5 min at 500g) until the supernatant becomes colorless. The washed core-shell particles were finally placed in absolute ethanol. Dried powder was obtained by slowly evaporating ethanol on a hot plate. The resulting product is hereafter named the as-prepared powder. Based on thermogravimetric analyses, a preliminary heat treatment at $600{ }^{\circ} \mathrm{C}$ under air was then carried out to completely remove all the organic residues. A short time of $1 \mathrm{~h}$ was used in order to minimize the morphology modification of the shell nanocrystallites. The resulting powder is hereafter named the pre-heated powder. 


\subsection{Sintering process}

The sintering process was performed on the pre-heated powder with SPS equipment (Dr Sinter SPS-2080 (Syntex Inc.) located at the Plateforme Nationale de Frittage Flash du CNRS, CIRIMAT Toulouse, France). Powder samples without any sintering aids were loaded into an $8 \mathrm{~mm}$ inner diameter graphite die. A sheet of graphitic paper was placed between the punch and the powder as well as between the die and the powder for easy removal. The treatment was conducted in a vacuum (residual cell pressure $<3 \mathrm{~Pa}$ ). A pulse configuration of 12 pulses (one pulse duration $3.3 \mathrm{~ms}$ ) followed by 2 periods $(6.6 \mathrm{~ms})$ of zero current was used. Heating rates of $150{ }^{\circ} \mathrm{C} \mathrm{min}^{-1}$ and

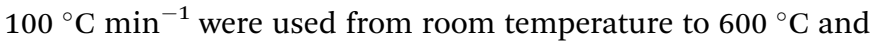
from 600 to $1100{ }^{\circ} \mathrm{C}$, respectively, where a 3 min dwell was applied, and then the samples were cooled down with a rate of $50{ }^{\circ} \mathrm{C} \mathrm{min}^{-1}$. An optical pyrometer, focused on a little hole at the surface of the die, was used to measure the temperature. A uniaxial load (corresponding to $50 \mathrm{MPa}$ ) was gradually applied during the last minute of temperature increase, maintained during the dwell and then gradually released during cooling.

A final heat treatment in air at $800{ }^{\circ} \mathrm{C}$ was carried out for removing the contamination on the pellet surface coming from graphite foil protection. Uncoated BT particles were also sintered under the same conditions in order to obtain reference ceramics for comparison and to better estimate the composite effect on the dielectric properties.

\section{Results and discussion}

\subsection{BT@MgO core-shell particles}

Fig. 1a shows the typical TEM micrograph obtained on the BT@MgO dispersion after a complete washing process. The main observation that should be underlined is that a distinct porous shell composed of nanoparticles less than $5 \mathrm{~nm}$ in size completely surrounds the BT particles (see also details in
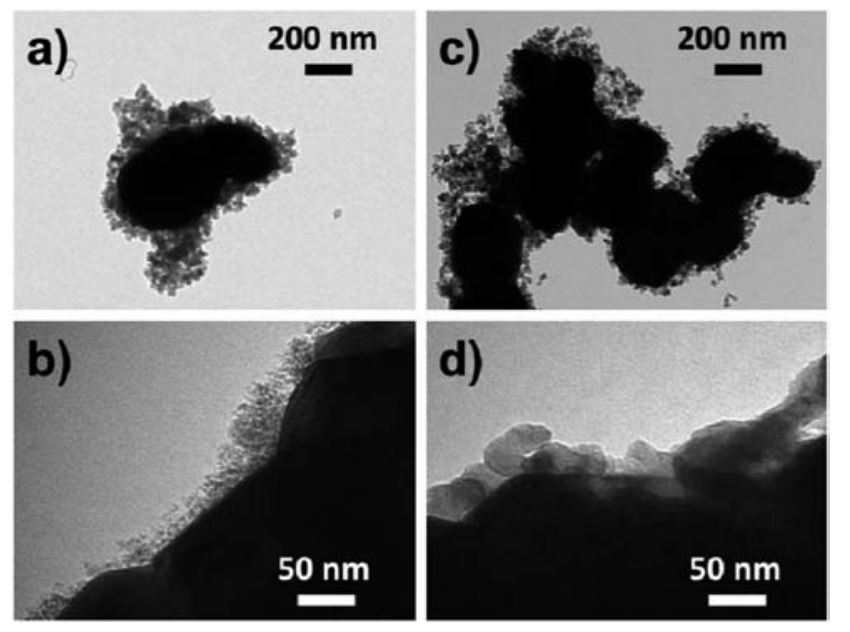

Fig. 1 Bright-field TEM micrographs of (a) the as-prepared BT@MgO core-shell nanoparticles with (b) an enlarged view of the interface region and (c) the pre-heated $\mathrm{BT}(\mathrm{MgO}$ core-shell particles with an enlarged view of the interface region (d).
Fig. 1b). The shell thickness is however not perfectly regular all around the particles with sometimes the presence of $\mathrm{MgO}$ nanoparticle bunches which remain attached on the BT particle surface despite all the washing sequences (as shown in Fig. 1a); it fairly varies around 15 to $50 \mathrm{~nm}$ with some thicker sections up to $150 \mathrm{~nm}$. XRD patterns obtained on the corresponding as-prepared dried powder clearly evidence the presence of $\mathrm{MgO}$ with its two main diffraction peaks unambiguously distinguishable from BT ones, in addition with large width confirming the nanosized character of the particles (Fig. 2a).

In order to deeply investigate this $\mathrm{MgO}$ outer shell with a chemical probing at the nanoscale, STEM-EDX has been carried out on a representative and non-aggregated core-shell particle with a complete $\mathrm{MgO}$ coating (Fig. 3). As barium and titanium concentrations are similar, only the second one is displayed and compared with the oxygen and magnesium amounts. The
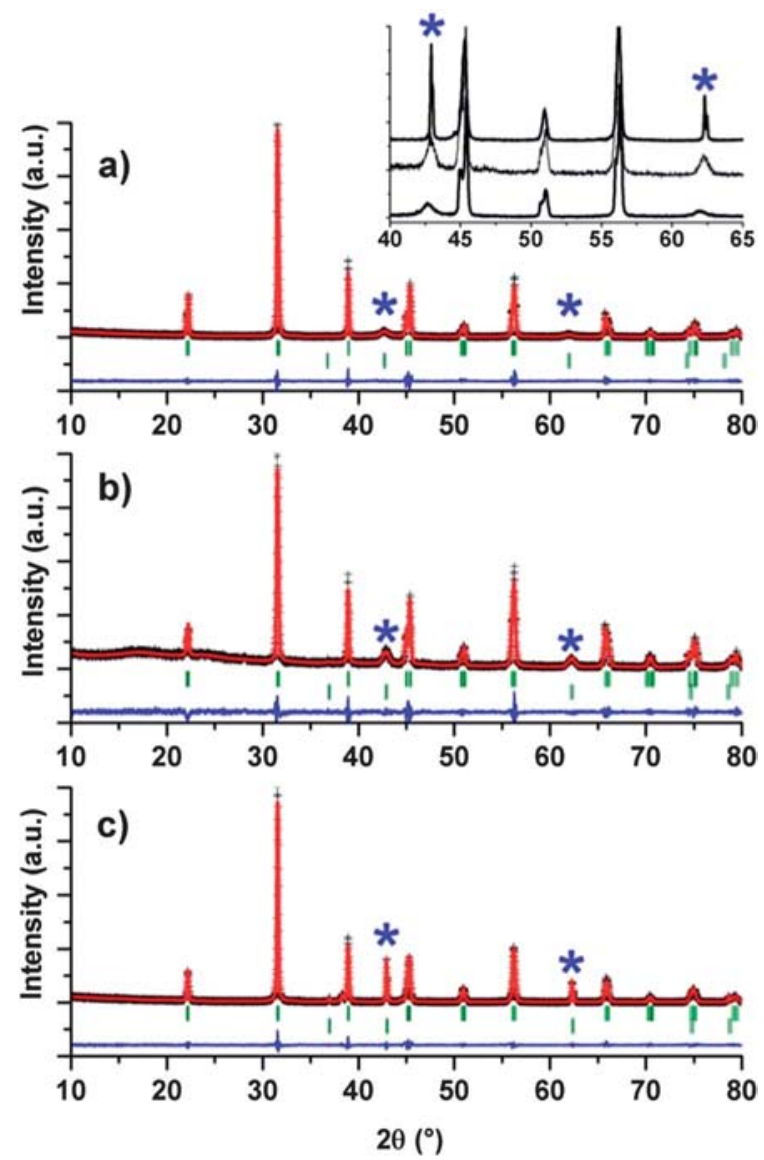

Fig. 2 XRD patterns of BT (aMgO obtained (a) after the coating process on the as-prepared dried powder, (b) on the pre-heated powder ( $1 \mathrm{~h}$ at $600{ }^{\circ} \mathrm{C}$ ), and (c) on a ground sintered ceramic. Observed, calculated (profile matching) and difference profiles as black crosses, red line and blue line, respectively. Bragg positions as vertical lines. Blue stars highlight the main diffraction peaks of $\mathrm{MgO}$ : (200) and (220) around $2 \theta$ $=43^{\circ}$ and $62^{\circ}$ respectively. Intensities are normalized by the highest diffraction peak. The top-right inset highlights the general decrease of the FWHM of the MgO diffraction peaks: from the as-prepared to the pre-heated and then to the sintered samples (from bottom to top, respectively). 


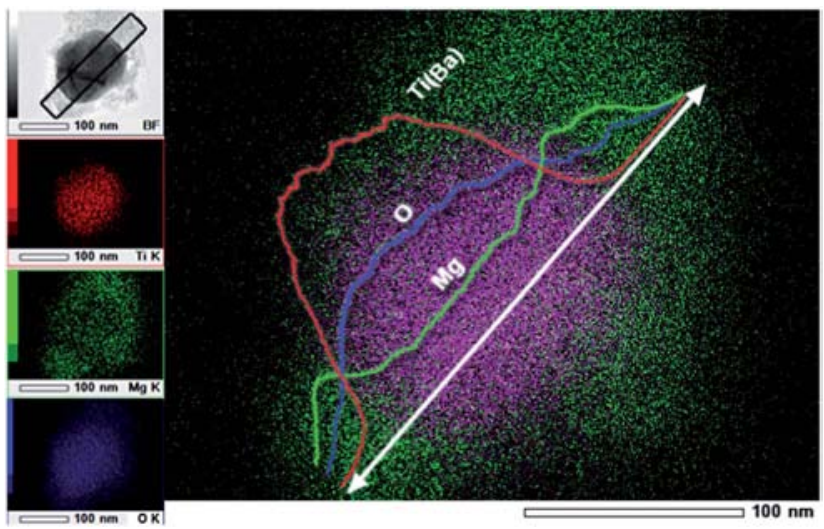

Fig. 3 STEM micrograph of a unique BT $\mathrm{AMgO}$ core-shell particle obtained from the as-prepared powder (a) and the corresponding chemical images drawn from the EDX map: for titanium in red (b), magnesium in green (c) and oxygen in blue (d). The barium concentration is not represented as it follows the titanium one in agreement with the BT chemical formula. The 3 -color resulting image (e) evidences that magnesium is present all around the BT core. This is especially highlighted by the linear profile of the magnesium concentration with two distinct maxima above the shell region.

resulting 3-color image highlights the peculiar magnesium localization all around the BT particle. Whereas TEM 2D images can sometimes mistakenly suggest a crown-like coating, the linear profile representing the magnesium amount evolution along a line that crossed through the coated particle evidences a $3 \mathrm{D}$ coating totally covering the spherical BT particle. Indeed two maxima are observed above the shell, while the concentration does not totally vanish above the BT core. The same nanostructuration of the MgO shell was also observed through HRTEM. An enlarged selected area showing the interface region between the BT core and the $\mathrm{MgO}$ shell is presented in Fig. 4. On the core side, BT reticular planes are clearly evidenced. As it has been confirmed by XRD (Fig. 2a), BT crystallizes in the tetragonal system (space group $P 4 \mathrm{~mm}$ ). From the estimation of the corresponding reticular distance, around $4.0 \AA$, it is therefore difficult to assign the observed planes to the (100) or (001) ones. On the shell side, the Fast Fourier Transform (FFT) of the whole area shows the occurrence of diffraction rings that can be indexed to the $\{111\},\{200\}$ and $\{220\}$ planes of a face-centered cubic phase structure in agreement with the $\mathrm{MgO}$ one. Moreover, these rather continuous rings coupled with the small monocrystalline domains observed on the left hand-side in Fig. 4 confirm the nanosized nature of the $\mathrm{MgO}$ particles randomly oriented within the shell.

A slight mass loss as well as a powder color change from light cream to white are observed after the preliminary heat treatment at $600{ }^{\circ} \mathrm{C}$ and confirmed that this step is mandatory to ensure a complete removal of all the organic residues before processing SPS. At this intermediate stage the core-shell morphology is checked by TEM. The corresponding micrograph presented in Fig. 1c shows that the MgO shell is still bound to BT particles. The overall thickness of the shell remains quite unchanged except the mean diameter of $\mathrm{MgO}$ grains that make-up the shell, which increases up to 10 to

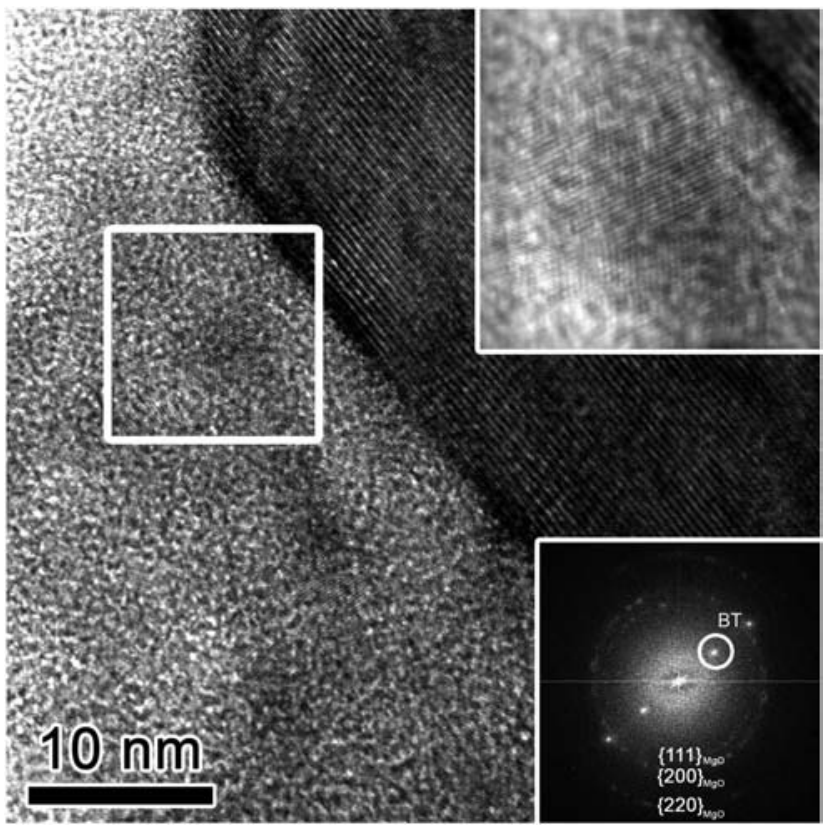

Fig. 4 HRTEM micrograph of the same BT@MgO particle as in Fig. 3 (from the as-prepared powder) with an enlarged view of the coreshell interface. The top-right inset is obtained after image processing (Wiener filtering) in order to underline the $\mathrm{MgO}$ reticular planes and to estimate the size of the $\mathrm{MgO}$ particles (mean diameter less than $10 \mathrm{~nm}$ ). The corresponding FFT image in the bottom-right inset shows the crystallized BT core while the diffraction rings confirm that the shell consists of aggregated and randomly oriented $\mathrm{MgO}$ nanocrystallites in the face-centered cubic phase structure.

$20 \mathrm{~nm}$ in diameter (Fig. 1c and d). In their studies, Kim et al. observed after thermal treatment of their metal alloy nanoparticles surrounded by $\mathrm{MgO}$ nanocrystals, the formation of a flower-like pattern over each nanoparticle constituted by distinct $\mathrm{MgO}$ crystals of $10 \mathrm{~nm}$ in size as "petals". ${ }^{22,23}$ With a mean diameter around $300 \mathrm{~nm}$, in the present study the BT particles are rather larger but the $\mathrm{MgO}$ particle size after the thermal treatment is in the same order of magnitude (10 to $20 \mathrm{~nm}$, see also the enlarged view in Fig. 1d). Considering the very small size of the initial $\mathrm{MgO}$ crystallites, lower than $5 \mathrm{~nm}$ in diameter, coalescence may occur at such a moderate temperature. In addition to the TEM analysis, the XRD powder pattern confirms that the crystallite growth is promoted as the full-width at half-maximum (FWHM) of the two MgO main diffraction peaks clearly decreases (Fig. 2).

From the ICP AES analysis, barium, titanium and magnesium concentrations were found to be around $28.4 \mathrm{mg} \mathrm{L}^{-1}$, $10.3 \mathrm{mg} \mathrm{L}^{-1}$ and $13.2 \mathrm{mg} \mathrm{L}^{-1}$, respectively. In consequence, the amount of $\mathrm{MgO}$ in the core-shell powder samples was found to be around $30.5 \mathrm{wt} \%$. This experimental value appears in rather good agreement with the initial amount of the magnesium precursor (i.e. $29.2 \%)$. Based on an ideal model of one spherical BT particle $(\varnothing=300 \mathrm{~nm}$ and with a mass density between 5.86 and $6.02 \mathrm{~g} \mathrm{~cm}^{-3}$ according to the literature) coated by a continuous and regular $\mathrm{MgO}$ shell $\left(\rho=3.58 \mathrm{~g} \mathrm{~cm}^{-3}\right)$, this $\mathrm{MgO}$ weight content involves a shell thickness around $30 \mathrm{~nm}$. From the TEM observations presented in Fig. 1 it is difficult to 
determine accurately the $\mathrm{MgO}$ shell thickness, but an average value of $30 \mathrm{~nm}$ seems quite reasonable.

As a first conclusion the alternative approach based on the thermolysis process enables successful coating of the ferroelectric particle by a complete $\mathrm{MgO}$ shell. The shell is directly composed of a multitude of nanosized and crystallized $\mathrm{MgO}$ particles displaying high surface energy and singular thermomechanical properties which will be beneficial thereafter.

\subsection{Discussion about the process and the mechanism of MgO coating}

The MgO shell morphology gives some indications that help the understanding of the coating process. For example, compared with silica coating onto ferroelectric particles, the homogeneous shell logically results in a seed-growth sol-gel process starting onto the particle surface with a thickness that regularly increases with the nominal precursor amount., ${ }^{5,10,11}$ In the present study, the preliminary TEM observations as well as the HRTEM analysis evidence a different mechanism. Indeed, the shell is not homogeneous as it consists of aggregated $\mathrm{MgO}$ particles with random orientations (Fig. 1, 3 and 4). Therefore the first sequence of the coating mechanism consists of the synthesis of $\mathrm{MgO}$ nanosized particles in solution. The magnesium precursor is first complexed by oleic acid. This organometallic precursor decomposes at high temperature leading to the formation of the metal oxide. In order to extract and wash the particles at the end of the reaction, the organophilic dispersion is mixed with ethanol, a non-solvent of oleyl ligands. When a lipophilic solvent, such as cyclohexane, is exclusively used to wash the particles, pieces of aggregated $\mathrm{MgO}$ particles and free $\mathrm{MgO}$ nanoparticles are unhooked from the BT particle surface (see Fig. S1 in the ESI $\dagger$ ). It is therefore necessary to carry out the washing sequence in ethanol as the major solvent and contain a partial amount of cyclohexane for the extraction of the residual organics (tetradecanediol and products resulting from the magnesium oleate decomposition). The centrifugation speed is another important parameter. Indeed, it has been empirically observed that fast centrifugation sequences (typically $5 \mathrm{~min}$ at $9000 \mathrm{~g}$ ) usually lead to an uncompleted $\mathrm{MgO}$ shell around the BT particles and more free $\mathrm{MgO}$ fragments that seem to be torn out from the BT surface. Consequently the centrifugation rate has been reduced to $500 \mathrm{~g}$ to obtain BT particles with a preserved $\mathrm{MgO}$ shell. In light of these observations, it can be concluded that the mechanism of shell formation mostly involves physico-chemical interactions (van der Waals adhesion, mainly London forces) which occur after the $\mathrm{MgO}$ particle formation. In addition, the presence of a large excess of $\mathrm{MgO}$ nanoparticles compared with the number of BT particles introduced (around $1.5 \times 10^{5}$ times assuming nanospheres of $5 \mathrm{~nm}$ in diameter for $\mathrm{MgO}$ and $300 \mathrm{~nm}$ for BT) is also responsible for such granular shell morphology. The use of a polar protic solvent, such as ethanol, causes the agglomeration of $\mathrm{MgO}$ nanoparticles which are present in large majority all around the ferroelectric particles. These weak adhesion forces can be broken by dissolution in good (non-polar) solvent or high-speed centrifugation.

\subsection{Sintering process and ceramic dielectric properties}

The SPS technique is a powerful tool to obtain dense materials within a very short sintering cycle. ${ }^{25,26}$ Therefore one of its main advantages is the conservation of the specific nanostructuration as it has been already shown for different architectures. ${ }^{11,13,14,27}$ The sintering was carried out on pre-heated powder samples, i.e. without any organic residues, and on uncoated BT powder for comparison. Fig. 5 shows a FEG-SEM micrograph obtained on the composite ceramic fracture. Sub-micrometric $\mathrm{MgO}$ grains are clearly visible instead of the nanocrystallites initially observed in the shell of the pre-heated powder (Fig. 1c and d). Such a size increase is in agreement with the narrow and intense $\mathrm{MgO}$ peak observed on the XRD pattern obtained after SPS (Fig. 2c). The initial core shell architecture is transformed in situ during the SPS process into a uniform distribution of submicrometric sized BT and MgO. Such a change in the morphology can be explained by considering (i) the variation of the shell thickness and the relatively weak cohesion of the $\mathrm{MgO}$ nanocrystallites in particular in regions the most distant from the surface of the cores, and (ii) the soft plastic behavior of crystalline $\mathrm{MgO}$ under the SPS conditions. Surprisingly there is no grain growth of the BT core, even when several grains, apparently free of $\mathrm{MgO}$, are in close contact. Such a grain growth limitation is highlighted by the comparison with both

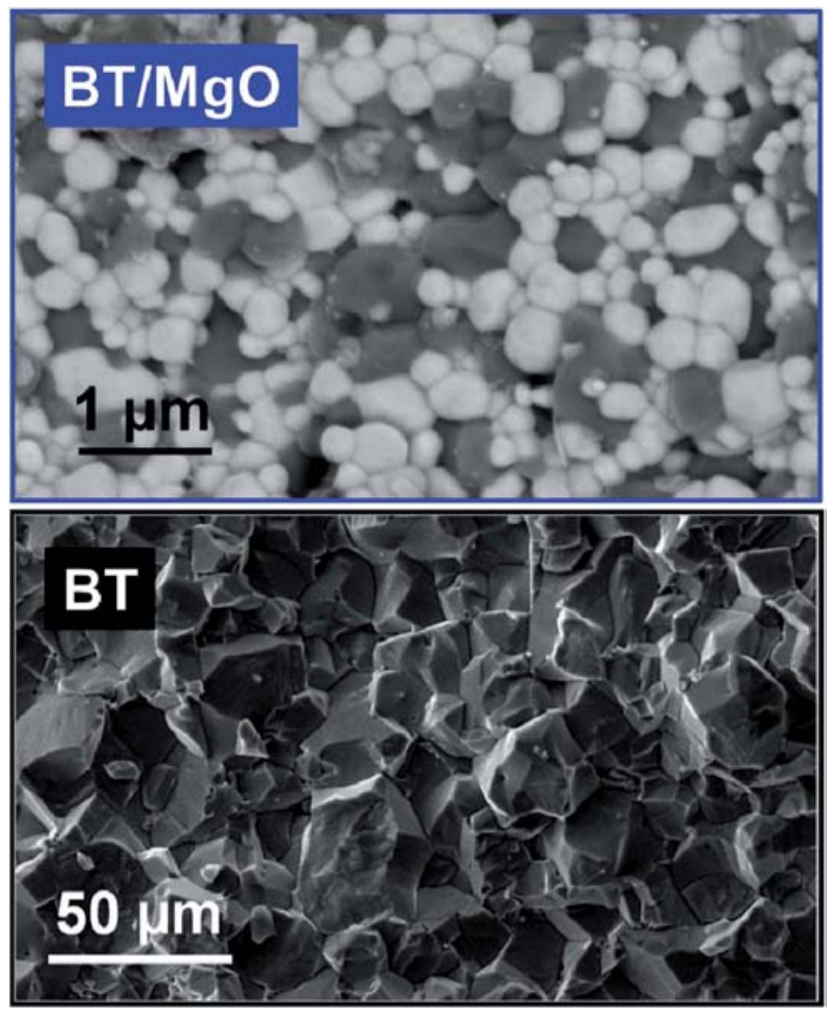

Fig. 5 (Top) The FEG-SEM micrograph in back-scattering electron mode of the fracture of the sintered composite ceramic shows the typical obtained nanostructure with $\mathrm{BT}$ and $\mathrm{MgO}$ randomly dispersed. (Bottom) The SEM micrograph of the uncoated BT ceramic highlights the BT grain growth during SPS which is not observed in the composite material, thanks to the impact of $\mathrm{MgO}$. 
uncoated BT ceramics for which the grain size increases up to more than $10 \mu \mathrm{m}$ during SPS (SEM micrograph in Fig. 5) and the very rare large BT grains observed within the sample and corresponding probably to uncoated grains during the synthesis. A pinning effect can hinder grain coarsening. However to explain such an efficient grain growth inhibition, an assumption could be the remaining in the final composite of nanosized $\mathrm{MgO}$ grains bound to the ferroelectric cores, and acting as a diffusion barrier efficiently preventing BT from grain boundary diffusion/ migration and grain growth. The intergranular fracture mode observed argues in favour of such an assumption. However TEM observations should be required to support this hypothesis.

Finally, the electron microscopy analysis reveals a low level of porosity, lower than $10 \%$, in good agreement with the relative density of at least $90 \%$ (from the measured volumetric mass of $4.73 \mathrm{~g} \mathrm{~cm}^{-3}$ and the estimation of $30.5 \mathrm{wt} \%$ content of $\mathrm{MgO}$ ). Reaching such a level of densification represents a good achievement in the field of nanostructured ceramics. ${ }^{4}$ The in situ dilatometry data collected during SPS are displayed in Fig. S2. $\dagger$ Around $600{ }^{\circ} \mathrm{C}$ a first jump in the displacement curve is observed for the BT-MgO composite but not for the BT reference. Even if the applied load is low at this stage of the SPS cycle (i.e. enough to ensure the electrical contact on the stack), this first phenomenon can be attributed to grain rearrangements by sliding due to the low cohesion between the $\mathrm{MgO}$ nanosized grains of the shell. This is a preliminary step of sub-micrometric $\mathrm{MgO}$ grain formation, as displayed in the schematic representation of the densification process (Fig. 6). The second phenomenon clearly evidenced is the onset of the densification around $950^{\circ} \mathrm{C}$ for the composite material, which is $100{ }^{\circ} \mathrm{C}$ lower than the densification temperature of the uncoated BT reference sintered under the same conditions. The main shrinkage corresponds to the densification of the $\mathrm{MgO}$ agglomerates located in between the BT grains. Considering the change in the yield stress of nanocrystallized $\mathrm{MgO}$ with temperature, the sintering conditions that are used for this study correspond to a domain where $\mathrm{MgO}$ may be plastically deformed. ${ }^{28}$ As a result enhanced densification kinetics assisted by plastic yield is expected in the composite ceramic. The major part of the sintering takes place during heating as shown by the shrinkage derivate maximum at $1000{ }^{\circ} \mathrm{C}$ (instead of $1060{ }^{\circ} \mathrm{C}$ for uncoated $\mathrm{BT})$. In the final stage of the sintering, coarsening of the nanosized $\mathrm{MgO}$ occurs by the diffusion process and grain boundary migration, leading to sub-micrometer particle size (Fig. 6).

The XRD powder pattern of the sintered composite ceramic is shown in Fig. 2c. In comparison with XRD patterns obtained from the as-prepared powder and after the heat treatment at 600 ${ }^{\circ} \mathrm{C}$, the FWHM of the MgO diffraction peaks is still decreasing, reflecting the $\mathrm{MgO}$ crystallite coarsening (inset of Fig. 2). The main outcome when comparing the XRD patterns, not only at the different stages of the composite elaboration but also with BT references, lies in the shape of the (002) and (200) diffraction peaks (see Fig. S3 $\dagger$ ). For both raw and sintered BT powders, they
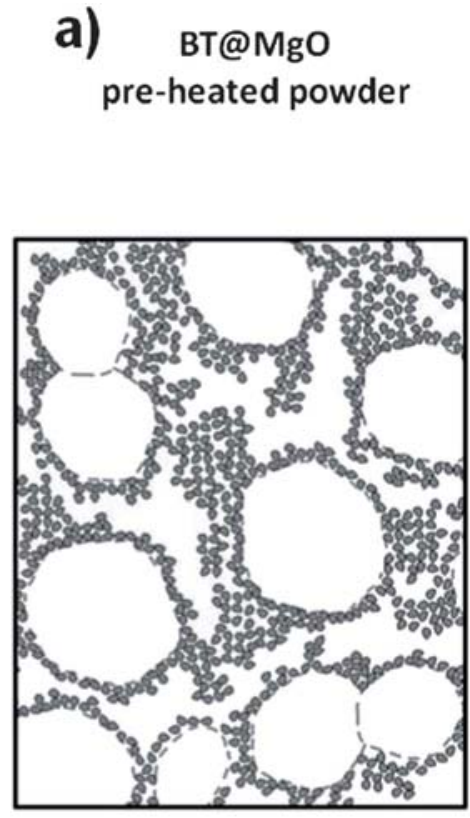

\section{b) BT@MgO compacted powder}

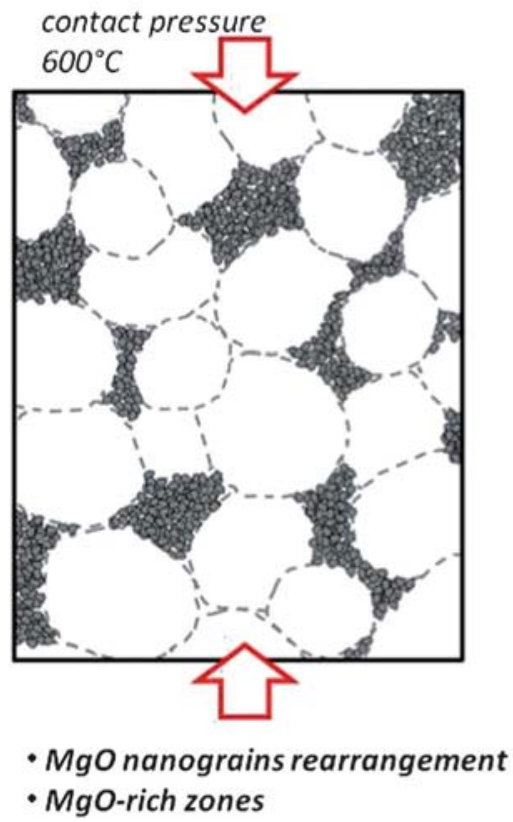

\section{C) $\mathrm{BT} / \mathrm{MgO}$ composite ceramic}

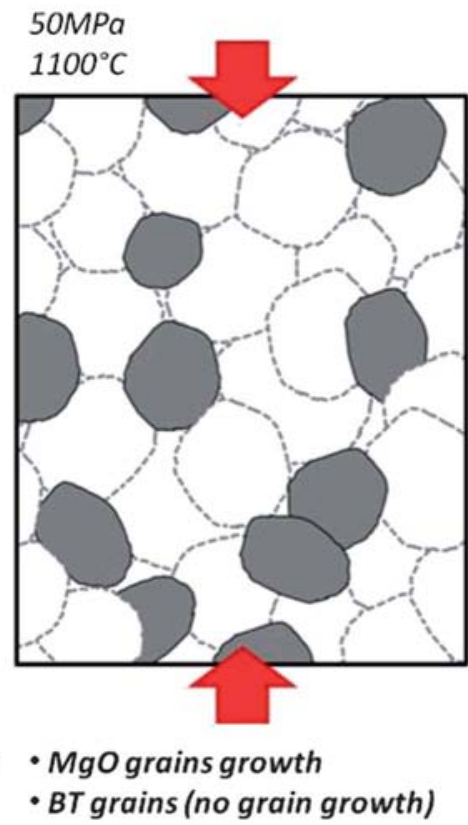

Fig. 6 Schematic drawing of the densification process occurring during the SPS. The nominal state (a) consists of BT@MgO core-shell particles. With the initial pressure at $600{ }^{\circ} \mathrm{C}$ applied in the SPS die (b), a rearrangement of the $\mathrm{MgO}$ occurs and local zones become MgO-rich. With the combine action of pressure and temperature (c), sub-micrometric-sized MgO grains are obtained from the nanocrystallite coarsening. The BT grain growth is avoided, thanks to the pinning effect of the surrounding $\mathrm{MgO}$. 
are clearly split in agreement with the tetragonal state (space group $P 4 \mathrm{~mm}$ ). The tetragonal state is still evidenced for the asprepared and the pre-heated BT@MgO powder, with however a slight change in the doublet profile which becomes more pronounced for the sintered composite ceramic with a barely visible tetragonal splitting. Such evolution in the XRD profile is similar to that usually observed when lowering the grain size and is linked to a decrease of the BT lattice tetragonality. ${ }^{29-31}$ In the present case, the grain size remains constant around $300 \mathrm{~nm}$ throughout the process; therefore, an additional contribution from the MgO has to be taken into account. The stress generated by the shell and/or by the MgO surrounding grains during the SPS cycle might be the additional effect. The thermal mechanical stress, arising in particular upon cooling, is due to the difference in the thermal expansion coefficient between $\mathrm{BaTiO}_{3}$ and $\mathrm{MgO}$. It is efficient because the ferroelectric grain size is close to the critical grain size towards the stabilization of the cubic state. In such a case even a moderate stress can trigger the change in the lattice symmetry.

The dielectric properties of the sintered composite and BT ceramics at $10 \mathrm{kHz}$ are displayed in Fig. 7. Specific features resulting from the composite effect can be underlined when considering the dielectric properties of the nanostructured composite ceramic. As expected the permittivity is strongly lowered compared to BT in the entire temperature range investigated. Such a decrease is associated with a significant thermal stabilization arising from the broadening and flattening of the permittivity anomaly: $30 \%$ of variation from the
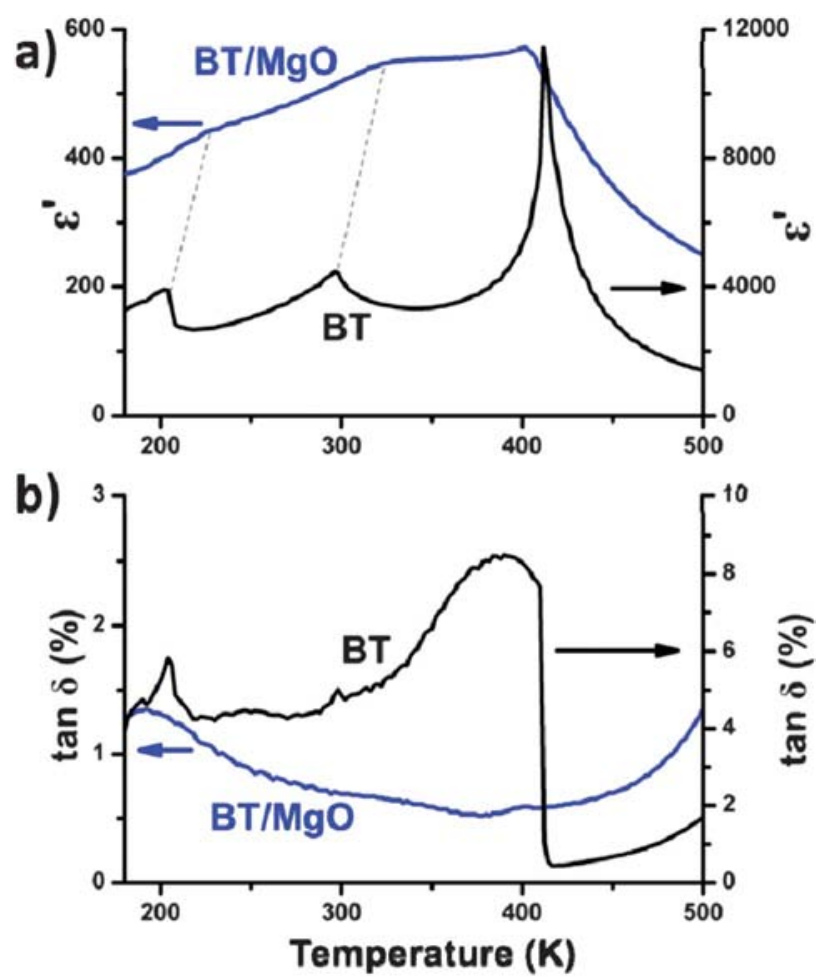

Fig. 7 Thermal variations of dielectric permittivity (a) and losses (b) at $10 \mathrm{kHz}$ for the composite and BT SPS ceramics (blue and black lines, respectively). A second scale is set for $\mathrm{BT}$ to help the comparison.
Curie temperature down to $200 \mathrm{~K}$ instead of $66 \%$ for uncoated BT. In the composite ceramic, the addition of the dielectric phase strongly impacts the Curie-Weiss temperature which is shifted down to $333 \mathrm{~K}$. The second effect is a drastic decrease of the dielectric losses which lies close to $0.7 \%$ at $10 \mathrm{kHz}$ without the strong variation associated with the Curie temperature. No dispersion occurs in the investigated frequency range. The three distinct maxima of permittivity as a function of temperature, characteristic of the structural transitions, are still observed but shifted compared to the obtained coarse grained BT ceramic: the cubic-tetragonal transition temperature is slightly decreased while those of tetragonal-orthorhombic and orthorhombic-rhombohedral are increased. One can note that the Curie temperature of uncoated BT is relatively high compared to average values reported in the literature. This can be explained by an increased mobility of domains arising from the very large grain size. ${ }^{32}$ Then we consider that the maximum of permittivity in the composite ceramic occurs in the expected temperature range for $300 \mathrm{~nm}$ grain size. This discards any interdiffusion between the two components as the origin of the shift in the Curie temperature. Regarding the evolution of the other structural transitions, they are believed to mainly result from the size/stress effect and are in good agreement with both thermodynamic simulations and reported experimental data. ${ }^{33}$ The stress generated on BT cores during the densification process by SPS can play a role in enhancing the stabilization of the rhombohedral phase. The modification of the Curie constant, from $1.38 \times 10^{5}$ in BT to $0.42 \times 10^{5}$ in the composite ceramic, is also assigned to a "stress effect" (see Fig. S4 $\dagger$ ). The overall dielectric characteristics of the composite ceramics can be understood by considering (i) the $300 \mathrm{~nm}$ size of the ferroelectric particles which is the borderline of the regime regarding the size effect (decrease of the tetragonality),,$^{\mathbf{3 0}, 31}$ and (ii) the role of the dielectric phase uniformly distributed among the ferroelectric grains which affects the dielectric response of the ferroelectric component both extrinsically as a composite effect and intrinsically. We ascribe the intrinsic effect to the stress generated during SPS sintering through the extended interfaces between the two components in all the directions. The modification of both the XRD profile and the Curie-Weiss parameters in the composite ceramic compared to the uncoated BT supports these conclusions. ${ }^{14}$

\section{Conclusions}

BT@MgO core-shell particles used as precursors for ferroelectric ceramics are successfully obtained by a thermal decomposition of organometallic precursors. Electron microscopy analyses enable characterization of the $\mathrm{MgO}$ shell morphology as a cluster of $\mathrm{MgO}$ nanosized crystallites with random orientations. The $\mathrm{MgO}$ shell is obtained by a self-assembly process involving weak adhesion forces. In the first stage of the sintering, the uniaxial pressure applied in the SPS die and the weak cohesion within the initial $\mathrm{MgO}$ shell allows reorganization of the nanocrystallites by grain sliding/rotation. Densification is first enhanced by plastic deformation and subsequent $\mathrm{MgO}$ grain growth occurs in the last stage of the sintering at high 
temperature. However grain growth of the ferroelectric cores is remarkably avoided, suggesting that the surrounding $\mathrm{MgO}$ particles act as an efficient diffusion barrier. As a result a uniform and dense nanostructure made of sub-micrometricsized $\mathrm{MgO}$ and $\mathrm{BT}$ grains is formed in situ during SPS. No signature of interdiffusion between $\mathrm{BT}$ and $\mathrm{MgO}$ was evidenced despite the high density of interfaces between the two components. However the stress generated during SPS through such extended interfaces strongly impacts the dielectric properties. A flattening of the permittivity values, associated with a decrease and thermal stabilization of the dielectric losses (around 0.7\%), is evidenced and the Curie-Weiss parameters are significantly modified compared to uncoated BT. Such results confirm the relevance of the approach combining the core-shell architecture and the SPS to obtain nanostructured composite ceramics with tailored dielectric properties.

An in-depth study of the close interface region between the ferroelectric core and the $\mathrm{MgO}$ shell at different stages of the processing is clearly mandatory to fully understand how the $\mathrm{MgO}$ nanocrystallites are bound to the BT particles, and to reach an accurate control of the $\mathrm{MgO}$ shell thickness. Such an interface investigation is in progress. In addition the flexibility of the soft plastic MgO particles under SPS conditions should be exploited by tuning the pressure cycle during the SPS process in order to control the nanostructure in such functional multimaterials.

Finally, the thermolysis process appears to be an efficient route to coat ferroelectric particles in a crystalline dielectric shell and the versatility of SPS in terms of "nanostructure design" is highlighted here to achieve dense composite ceramics. The simultaneous improvement of the synthesis, the shaping and the properties is still challenging in multi-materials. The promising results presented in this work should open the way to further controlled coating on different oxides towards new functional ceramics with tailored properties.

\section{Acknowledgements}

The Bordeaux Imaging Center (BIC, University of Bordeaux Segalen) is acknowledged for the TEM facility. This work has been made possible by the financial support of Conseil Régional d'Aquitaine and the ANR project ARCHIFUN (ANR-12-BS08-009).

\section{Notes and references}

1 W. Schärtl, Nanoscale, 2010, 2, 829.

2 S. Wei, Q. Wang, J. Zhu, L. Sun, H. Lin and Z. Guo, Nanoscale, 2011, 3, 4474.

3 R. G. Chaudhuri and S. Paria, Chem. Rev., 2012, 112, 2373.

4 M. T. Buscaglia, M. Viviani, Z. Zhao, V. Buscaglia and P. Nanni, Chem. Mater., 2006, 18, 4002.

5 S. Mornet, C. Elissalde, O. Bidault, F. Weill, E. Sellier, O. Nguyen and M. Maglione, Chem. Mater., 2007, 19, 987.

6 A. Bassano, V. Buscaglia, M. Sennour, M. Buscaglia, M. Viviani and P. Nanni, J. Nanopart. Res., 2010, 12, 623.

7 G. Evans, G. V. Duong, M. J. Ingleson, Z. Xu, J. T. A. Jones, Y. Z. Khimyak, J. B. Claridge and M. J. Rosseinsky, Adv. Funct. Mater., 2010, 20, 231.
8 C. Huber, M. Treguer-Delapierre, C. Elissalde, F. Weill and M. Maglione, J. Mater. Chem., 2003, 13, 650.

9 V. Hornebecq, C. Huber, M. Maglione, M. Antonietti and C. Elissalde, Adv. Funct. Mater., 2004, 14, 899.

10 S. Mornet, C. Elissalde, V. Hornebecq, O. Bidault, E. Duguet, A. Brisson and M. Maglione, Chem. Mater., 2005, 17, 4530.

11 U.-C. Chung, C. Elissalde, F. Mompiou, J. Majimel, S. Gomez, C. Estournès, S. Marinel, A. Klein, F. Weill, D. Michau, S. Mornet and M. Maglione, J. Am. Ceram. Soc., 2010, 93, 865.

12 L. C. Sengupta and S. Sengupta, Mater. Res. Innovations, 1999, 2, 278.

13 C. Elissalde, M. Maglione and C. Estournès, J. Am. Ceram. Soc., 2007, 90, 973.

14 U.-C. Chung, C. Elissalde, M. Maglione, C. Estournès, M. Pate and J. P. Ganne, Appl. Phys. Lett., 2008, 92, 042902.

15 W. Liao, R. Liang, G. Wang, F. Cao and X. Dong, Appl. Phys. Lett., 2011, 99, 202905.

16 H. Tian, J. Qi, Y. Wang, H. Chan and C. Choy, Prog. Solid State Chem., 2005, 33, 207.

17 H. Y. Tian, J. Q. Qi, Y. Wang, J. Wang, H. L. W. Chan and C. L. Choy, Nanotechnology, 2005, 16, 47.

18 J. Q. Qi, H. Y. Tian, Y. Wang, G. K. H. Pang, L. T. Li and H. L. W. Chan, J. Phys. Chem. B, 2005, 109, 14006.

19 W. Chen, H. Tian, J. Qi, Y. Wang, Z. Shen, X. Ruan and H. L. W. Chan, Key Eng. Mater., 2007, 334-335 II, 1037.

20 J. S. Park and Y. H. Han, J. Eur. Ceram. Soc., 2007, 27, 1077.

21 S. Liu, X. Li, H. Ji, Q. Jia and Y. Zhou, J. Mater. Sci.: Mater. Electron., 2012, 23, 1057.

22 J. Kim, C. Rong, Y. Lee, J. P. Liu and S. Sun, Chem. Mater., 2008, 20, 7242.

23 J. Kim, C. Rong, J. P. Liu and S. Sun, Adv. Mater., 2009, 21, 906.

24 M. T. Buscaglia, V. Buscaglia, L. Curecheriu, P. Postolache, L. Mitoseriu, A. C. Ianculescu, B. S. Vasile, Z. Zhe and P. Nanni, Chem. Mater., 2010, 22, 4740.

25 F. Maglia, I. G. Tredici and U. Anselmi-Tamburini, J. Eur. Ceram. Soc., 2013, 33, 1045.

26 J. F. Li, K. Wang, B. P. Zhang and L. M. Zhang, J. Am. Ceram. Soc., 2006, 89(2), 706.

27 T. Hungria, J. Galy and A. Castro, Adv. Eng. Mater., 2009, 11, 615.

28 R. Chaim, R. Marder, C. Estournès and Z. Shen, Adv. Appl. Ceram., 2012, 111, 280.

29 C. Valot, N. Floquet, M. Mesnier and J. C. Niepce, J. Phys. IV, 1996, 6(C4), 71.

30 T. Nagai, K. Iijima, H. J. Hwang, M. Sando, T. Sekino and K. Niihara, J. Am. Ceram. Soc., 2000, 83, 107.

31 Z. Zhao, V. Buscaglia, M. Viviani, M. T. Buscaglia, L. Mitoseriu, A. Testino, M. Nygren, M. Johnsson and P. Nanni, Phys. Rev. B: Condens. Matter Mater. Phys., 2004, 70, 024107.

32 B. Cheng, M. Gabbay, M. Maglione and G. Fantozzi, J. Electroceram., 2003, 10, 5.

33 S. Lin, T. Lü, C. Jin and X. Wang, Phys. Rev. B: Condens. Matter Mater. Phys., 2006, 74, 134115. 